\title{
Link Between Cyberbullying Victimization and Perpetration Among Undergraduates: Mediating Effects of Trait Anger and Moral Disengagement
}

This article was published in the following Dove Press journal: Psychology Research and Behavior Management

\author{
Guangbo Dou' \\ Ying Xiang ${ }^{2}$ \\ Xiaohua $\operatorname{Sun}^{3 *}$ \\ Liang Chen ${ }^{3, *}$ \\ 'College of Kinesiology, Shenyang Sport \\ University, Shenyang City, Liaoning \\ Province, People's Republic of China; \\ ${ }^{2}$ Department of Psychiatry, Dalian \\ Rehabilitation Center, Dalian City, \\ Liaoning Province, People's Republic of \\ China; ${ }^{3}$ School of Marxism, University of \\ Science and Technology Liaoning, Anshan \\ City, Liaoning Province, People's Republic \\ of China
}

*These authors contributed equally to this work
Purpose: Based on the general strain theory and moral engagement theory, this study aimed to explore the prediction of cyberbullying victimization on cyberbullying perpetration among college students and the mediating effect of trait anger and moral disengagement.

Materials and Methods: Data collected from 1183 college students $(M=20.62, S D=$ 1.21) from a university in Liaoning Province (China) were analyzed using the Cyberbullying Victimization Scale, Trait Anger Scale, Moral Disengagement Scale, and the Cyberbullying Questionnaire.

Results: There was a significant positive correlation between cyberbullying victimization, trait anger, moral disengagement, and cyberbullying perpetration. Cyberbullying victimization also predicted college students cyberbullying perpetration through the mediating effects of trait anger and moral disengagement. The mediating effects included two paths, which were the separate mediating effect of moral disengagement and the chain mediating effect of trait anger-moral disengagement. The total mediating effect is $6.15 \%$.

Conclusion: Trait anger and moral disengagement play a mediating role in the relationship between cyberbullying victimization and cyberbullying perpetration among college students. Keywords: college students, cyberbullying victimization, trait anger, moral disengagement, cyberbullying perpetration

\section{Introduction}

With the popularization of the Internet and the rapid development of information technology, cyberbullying perpetration as a new type of attack has become common and is being widely studied. Extensive research has examined the various forms of cyberbullying, and cyberbullying perpetration is generally defined as an aggressive, intentional act carried out by a group or an individual, using electronic forms of contact, repeatedly and over time against a victim who cannot easily defend themselves. ${ }^{1}$ Menesini and colleagues categorized two types of cyberbullying: visual and written-verbal behaviors, ${ }^{2}$ with previous research showing that visual behaviors were more severe than written and verbal acts. ${ }^{3,4}$ Although cyberbullying perpetration is most prevalent among early teens (11 to 14 years old), it can begin in middle school and continue into the college period, with the two modalities of cyberbullying remaining consistent. ${ }^{5,6} \mathrm{Zhu}$ and colleagues found that college students' cyberbullying perpetrators accounted for $17.32 \%$ of the students surveyed while cyberbullying victims accounting for $36.27 \%$ of the students surveyed, with both perpetrators and victims representing $46.41 \%$ of the students surveyed. ${ }^{7}$ Gibb
Correspondence: Xiaohua Sun; Liang

Chen

Tel +86-04 I2-5928008;

$+86-04$ I 2-5929598

Email sunxh@ustl.edu.cn;

chenlfly@ustl.edu.cn 
and Devereux (2014) conducted a survey among college students and found that cyberbullying victims accounted for $52 \%$ of the students surveyed. Cyberbullying victims are likely to vent their emotions through cyberbullying and become perpetrators themselves, contributing to a vicious cycle. ${ }^{8}$ Compared to traditional bullying, cyberbullying is characterized by anonymity, being spatiotemporal, and entails strong propagation, impacting the physical and psychological health of the victims (eg, loneliness, ${ }^{9}$ suicidal ideation ${ }^{10}$ ). Due to the potential extent of harm from cyberbullying, it has become a worldwide public health concern and received the attention of researchers and educators. Therefore, it is of immense theoretical significance to understand the causes of cyberbullying perpetration and to investigate the psychological mechanisms underlying victims behavior and the subsequent transformation to functioning as perpetrators.

\section{The Relationship Between Cyberbullying Victimization and Perpetration}

In the network environment, the roles of the bully and the victim can interchange. ${ }^{11}$ Similarly, the general strain theory (GST) has been adopted by researchers to explain the relationship between cyberbullying victimization and perpetration. ${ }^{12}$ According to GST, cyberbullying victimization, as a negative stimulus, is an important source of pressure leading to cyberbullying perpetration. ${ }^{13}$ Some studies have found that many victims of cyberbullying are also perpetrators of cyberbullying. ${ }^{14-16}$ Wong and colleagues conducted a study with adolescents in Hong Kong and found a significant positive correlation between cyberbullying victimization and perpetration. ${ }^{17}$ Cross-sectional and longitudinal studies have found that cyberbullying victimization significantly predicts cyberbullying perpetration, ${ }^{11,18}$ suggesting that cyberbullying victims are likely to transform into perpetrators.

\section{Mediating Effect of Trait Anger}

According to GST, a negative stimulus, as a stressor, leads to negative emotions, such as anger and frustration, and can result in violent or deviant behaviors as a form of coping strategy that individuals may use under stress in response to those negative emotions. ${ }^{19,20}$ In other words, cyberbullying is a coping strategy that individuals experiencing stress and strain may commit. Therefore, being bullied online could lead to negative emotional experiences, such as anger, that increase one's likelihood of engaging in cyberbullying perpetration. ${ }^{6}$ A study of college students indicated that cyberbullying victimization could directly and significantly predict cyberbullying perpetration and indirectly predict cyberbullying as an expression of anger. ${ }^{11}$ Spielberger further categorized anger as state anger and trait anger. ${ }^{21}$ Wang and colleagues surveyed 464 college students and found that trait anger is significantly positively correlated with cyberbullying perpetration. $^{22}$

\section{Mediating Role of Moral Disengagement}

In addition to emotional factors, cognitive factors play an important role in the relationship between cyberbullying victimization and cyberbullying perpetration. According to moral disengagement theory (MDT), individuals reduce psychological guilt and self-accusation through the mechanism of moral disengagement when displaying external problematic behaviors. ${ }^{23}$ Moral disengagement includes certain cognitive tendencies, such as redefining one's behavior to make it less harmful, minimizing one's responsibility for the consequences of one's behavior, and reducing identification with a victim's pain. ${ }^{24}$ As an important component of moral cognition, both traditional bullying and offline bullying, ${ }^{25}$ can indirectly affect the cyberbullying of teenagers through moral disengagement. Similarly, moral disengagement is also affected by emotional factors. Wang and colleagues found that moral disengagement mediated the relationship between trait anger and college students' cyberbullying perpetration. ${ }^{22}$

The mechanism by which cyberbullying victimization influences cyberbullying perpetration has previously been explored; therefore, this study aimed to better understand the mediating roles of trait anger and moral disengagement in the relationship between cyberbullying victimization and cyberbullying perpetration among Chinese college students based on GST and MDT. Specifically, this study tested four hypotheses. Hypothesis 1 stated that cyberbullying victimization would positively predict cyberbullying perpetration. Hypothesis 2 was that trait anger would mediate the relationship between cyberbullying victimization and college students' cyberbullying perpetration. Hypothesis 3 stated that moral disengagement would act as a separate mediator of the relationship between cyberbullying victimization and perpetration. Hypothesis 4 was that trait anger and moral disengagement would play a chain mediating role in the relationship between cyberbullying victimization and perpetration among college students. Figure 1 presents the proposed multiple mediation model. 


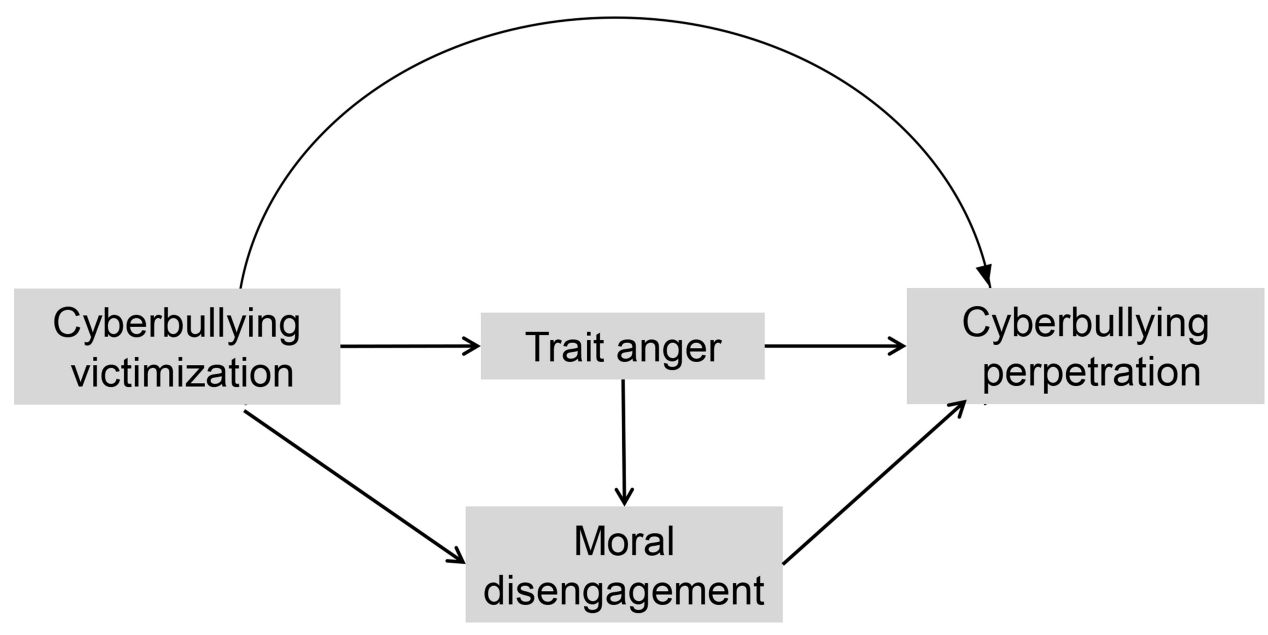

Figure I Assumption model of this study.

\section{Materials and Methods}

\section{Participants}

All participants provided their informed consent for inclusion before they participated in the study. The study was conducted in accordance with the Declaration of Helsinki, and the protocol was approved by the Research Ethics Committee of the University of Science and Technology Liaoning (China). The effect size was computed using $\mathrm{f}^{2}(\alpha=0.05$, one tail), and the total sample size was 1084 . Using random cluster sampling, 1200 college students were selected from a university in Liaoning Province to complete the questionnaires, and 1183 responses were valid. Informed consent was obtained from the participants. The students' age ranged from 17 to 23 years old, with an average age of 20.62 years $(S D=1.21)$. The sample consisted of 682 male $(57.7 \%)$ and 501 female students $(42.3 \%)$. Eight hundred and twenty-nine students were from the field of engineering $(70.1 \%), 155$ students were majoring in science (13.1\%), and 199 in liberal arts (16.8\%).

\section{Measures}

\section{The Cyberbullying Victimization Scale}

Erdur and Kavsut $^{26}$ developed the Cyberbullying Victimization Scale (CVS), which was validated by Zhou et al. ${ }^{27}$ The scale consists of 18 items (eg, "receiving hurtful emails") and uses a four-point rating scale ( $1=$ never met, $4=$ more than 5 times). The higher the total score, the more frequently the individual was bullied online. In this sample, the cronbach's $\alpha$ was 0.848 .

\section{State-Trait Anger Scale}

The State-trait Anger Scale was developed by Spielberger ${ }^{21}$ and validated by Luo et al. ${ }^{28}$ The scale constitutes of 10 items, including two subscales of temperamental trait anger (four items; eg, "I have a fiery temper") and reactive trait anger (six items; eg, "When I get frustrated, I want to hit someone"). It uses the four-point rating scale, ranging from 1 (never) to 4 (always), with higher scores indicating higher levels of trait anger in college students. For this study, the Cronbach's $\alpha$ coefficient of the total scale was 0.887 . The cronbach's $\alpha$ coefficients of the temperamental and reactive trait anger subscales were 0.892 and 0.802 , respectively.

\section{Moral Disengagement Scale}

Bandura et al formulated the moral disengagement scale (MDS) ${ }^{24}$ The items of the scale were designed for children and adolescents. To ensure its applicability with college students, Detert et al modified the scale. ${ }^{29}$ In this study, the Chinese version of the MDS that was validated by Wang and Yang was used. ${ }^{30}$ The scale contains eight dimensions: moral justification (four items; eg, "It's alright to fight to protect your friends"), euphemistic labeling (three items; eg, "It's okay to indulge once in a while"), advantageous comparison (three items; eg, "Compared with those who cheat in exams, not working hard is not a big deal"), displacement of responsibility (three items; eg, "If a person is forced to do something, this person is not to blame"), diffusion of responsibility (four items; eg, "Team members should not be blamed for mistakes made by the team"), distortion of consequences (three items; eg, "Making fun of people doesn't really hurt them"), attribution of blame (three items; eg, "A person who has been abused must have done something to deserve to be treated like this"), and dehumanization (three items; eg, "Those annoying people don't deserve to be treated as human beings"), totaling 26 items rated on a five-point scale $(1=$ strongly disagree, $5=$ strongly agree). Higher scores indicated higher levels of moral disengagement. For this study, the Cronbach's $\alpha$ coefficient 
was 0.915 , and the cronbach's $\alpha$ coefficients of the eight subscales ranged from 0.646 to 0.784 .

\section{The Cyberbullying Scale}

The Chinese version of the cyberbullying scale was created by Wright $^{31}$ and validated by Wang. ${ }^{32}$ The questionnaire includes two dimensions of direct cyberbullying perpetration (five items; eg, "Do you often spread rumors about your peers online or via text messages") and indirect cyberbullying perpetration (four items; eg, "Do you often instigate someone to antagonize your peers online or via text message"), totaling of nine items. Items are rated on a 5-point scale ranging from 1 (never) to 5 (always). The higher the score, the higher is the frequency of cyberbullying perpetration. For this study, the cronbach's $\alpha$ coefficient was 0.861 , with cronbach's $\alpha$ coefficients for direct and indirect cyberbullying perpetration being 0.799 and 0.815 , respectively.

\section{Statistical Processing and Analytic Plan}

In this study, SPSS 23.0 statistical software (SPSS, Inc, Chicago, IL, USA) was used to analyze descriptive statistics, reliability analysis, correlation analysis; Mplus 8.0 was selected for confirmatory factor analysis and the structural equation modeling. ${ }^{33}$ Correlational analyses tested the concurrent associations between the four variables. A confirmatory factor analysis was subsequently conducted to identify the factor loading of each item on the CVS. Structural equation modeling was conducted to test Hypotheses 1 to 4 . The goodness-of-fit indices were used included the Tucker-Lewis Index (TLI), the Comparative Fit Index (CFI), the Root-MeanSquare Error of Approximation (RMSEA), and the Standardized Root-Mean-Square Residual (SRMR). Missing values were replaced with the series mean method in SPSS 23.0.

\section{Results}

Correlation Between Cyberbullying Victimization, Trait Anger, Moral Disengagement, and Cyberbullying

\section{Perpetration}

Descriptive statistics and Pearson correlation analysis were conducted for cyberbullying victimization, trait anger, moral disengagement, and cyberbullying perpetration. Table 1 shows the means and standard deviations for the primary variables, and Table 2 is a correlation matrix of these variables. Significant positive correlations were found between the four variables.

\section{Testing the Mediating Effect}

Cyberbullying victimization was the independent variable, trait anger and moral disengagement were the mediating variables, and cyberbullying perpetration was the dependent variable in the model. Since the CVS contained many items, the study categorized the 18 items of the CVS according to the item packaging theory to simplify the measurement structure. ${ }^{34}$ A single dimension of confirmatory factor analysis was conducted on the 18 items. The items were packed according to the balance of the factor loading scores of each item package. Therefore, the items were categorized into three item-packages as the observational indicators of the CVS.

Mplus 8.0 was used to construct the structural equation model. The path significance for each hypothesized association in the models and the variance explained $\left(R^{2}\right)$ for each path were examined. The Total Effect Model A of cyberbullying victimization on cyberbullying perpetration was established, and the total effect and its significance were tested with Figure 2 showing the standardized path coefficients and significance. The results indicated that the total effect of cyberbullying victimization on cyberbullying perpetration was 0.642 , the total effect coefficient was significant $(p<0.001)$, and the fitting indexes were good (see Table 3 ). Cyberbullying perpetration was predicted by cyberbullying victimization $(p<$ 0.001 ), explaining $41.2 \%$ of the variance. Second, the significance of the path coefficient was examined sequentially. In this study, cyberbullying victimization was the independent variable, college students' cyberbullying perpetration the dependent variable, and trait anger and moral disengagement were the mediating variables. Subsequently, Mediating Model B was established (see Figure 3). Structural equation model analysis showed that all fitting indexes were good except TLI $<$ 0.90 and RMSEA $>0.080$ (see Table 3), indicating that the model was acceptable. The path coefficient of trait anger to cyberbullying perpetration was not significant $(r=0.051, p>$ $0.05)$. Cyberbullying perpetration was predicted by cyberbullying victimization $(p<0.001)$ and moral disengagement $(p<$ 0.01 ), which explained $34.8 \%$ and $1.99 \%$ of the variance, respectively. Therefore, the mediating role included two

Table I Descriptive Statistics of Variables $(N=1183)$

\begin{tabular}{|l|l|l|}
\hline Variables & $\mathbf{M}$ & SD \\
\hline Cyberbullying victimization & 1.14 & 0.22 \\
Trait anger & 1.65 & 0.48 \\
Moral disengagement & 1.57 & 0.50 \\
Cyberbullying perpetration & 1.07 & 0.24 \\
\hline
\end{tabular}


Table 2 Correlation Matrix Between Variables $(N=1 \mid 83)$

\begin{tabular}{|l|l|l|l|l|}
\hline Variables & $\mathbf{I}$ & $\mathbf{2}$ & $\mathbf{3}$ & $\mathbf{4}$ \\
\hline I Cyberbullying victimization & $\mathrm{I}$ & & & \\
2 Trait anger & $0.246^{* * *}$ & $\mathrm{I}$ & & \\
3 Moral disengagement & $0.245^{* * * *}$ & $0.477^{* * *}$ & $\mathrm{I}$ & \\
4 Cyberbullying perpetration & $0.522^{* * *}$ & $0.250^{* * *}$ & $0.277^{* * *}$ & $\mathrm{I}$ \\
\hline
\end{tabular}

Note: $* * * p<0.001$.

paths, the separate mediating role of moral disengagement and the chain mediating role of trait anger-moral disengagement.

The bias correction non-parametric percentile bootstrap method was used to test the mediating effect of the latent variable model. The sample was repeated 1000 times, and the $95 \%$ confidence interval was obtained. The results indicated that the mediating effect includes two indirect effects. First, the standardized indirect effect produced by the path from cyberbullying victimization to moral disengagement to cyberbullying perpetration. Its $95 \%$ confidence interval is 0.006 and 0.049 , excluding 0 (see Table 4). The mediating effect was 0.018 (see Table 4), accounting for $2.88 \%$ of the total effect. Second, the standardized indirect effect produced by the path from cyberbullying victimization to trait anger to moral disengagement to cyberbullying perpetration, with a $95 \%$ confidence interval of $(0.008,0.047)$, excluding 0 (see Table 4), and a mediating effect of 0.021 (see Table 4), accounting for $3.27 \%$ of the total effect. Therefore, the total mediating effect was $6.15 \%$.

\section{Discussion}

\section{The Relationship Between Cyberbullying Victimization and College Students Cyberbullying Perpetration}

Hypothesis 1 of this study proposed that cyberbullying victimization would positively predict cyberbullying perpetration. The result found that cyberbullying victimization has a significantly positive prediction on cyberbullying perpetration, supporting Hypothesis 1.
This result is consistent with previous research. ${ }^{11}$ According to GST, cyberbullying victimization, as a source of stress, can trigger a bullied person's negative emotions, such as anger. ${ }^{13}$ The previous study found that feelings of anger were more likely to foster engagement in cyberbullying. ${ }^{35}$ Indeed, research has shown that victims' feelings of negative emotion can reduce the effectiveness of their problem-solving abilities. ${ }^{19,20}$ Due to the anonymity of the network environment, bullied individuals are more likely to vent their negative emotions on the network, increasing their likelihood of becoming cyberbullying perpetrators. ${ }^{11,36,37}$

\section{Mediating Role of Trait Anger and Moral Disengagement}

Hypothesis 2 of this study proposed that trait anger would mediate the relationship between cyberbullying victimization and cyberbullying perpetration. Contrary to Hypothesis 2, this study found that trait anger did not act as a direct mediator but was an indirect mediator through moral disengagement. This finding supported Hypothesis 4 that trait anger and moral disengagement would create a mediating pathway in the relationship between cyberbullying victimization and perpetration. This is inconsistent with previous research, which has indicated that anger from being cyber-bullied increases an individual's likelihood of attacking others through the Internet. $^{11,22}$ Due to the anonymity of the Internet, many victims of cyberbullying do not know the true identity of the attackers; thus, they vent their anger by attacking others, thus creating a vicious cycle of cyberbullying. Although attackers typically modify their self-representation and conceal their real identity in the virtual world, they are less likely to be negatively evaluated or attacked by others. ${ }^{38,39}$ Furthermore, such cyberattacks on others could also trigger negative selfevaluation or negative emotions such as self-accusation and guilt. Consequently, a cyber attacker may reduce negative selfevaluation or negative emotions through moral

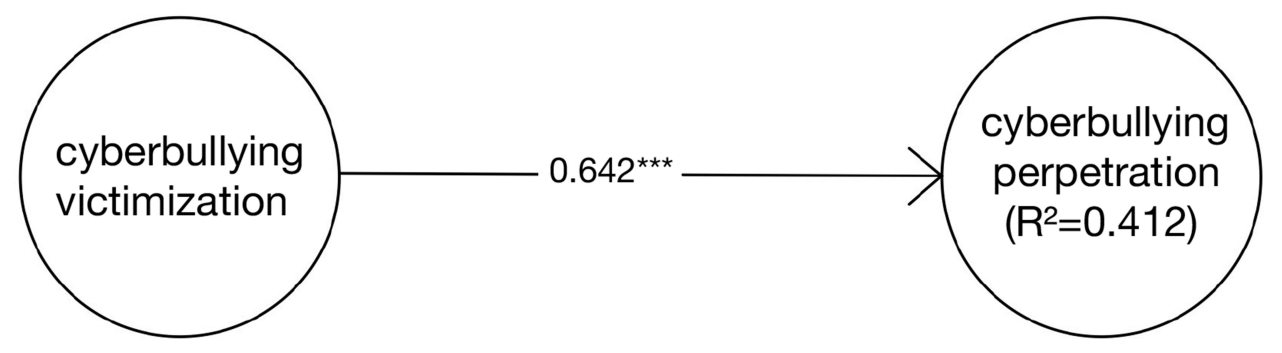

Figure 2 The model $\mathrm{A}$ of the total effect. Note: $* * * p<0.001$. 


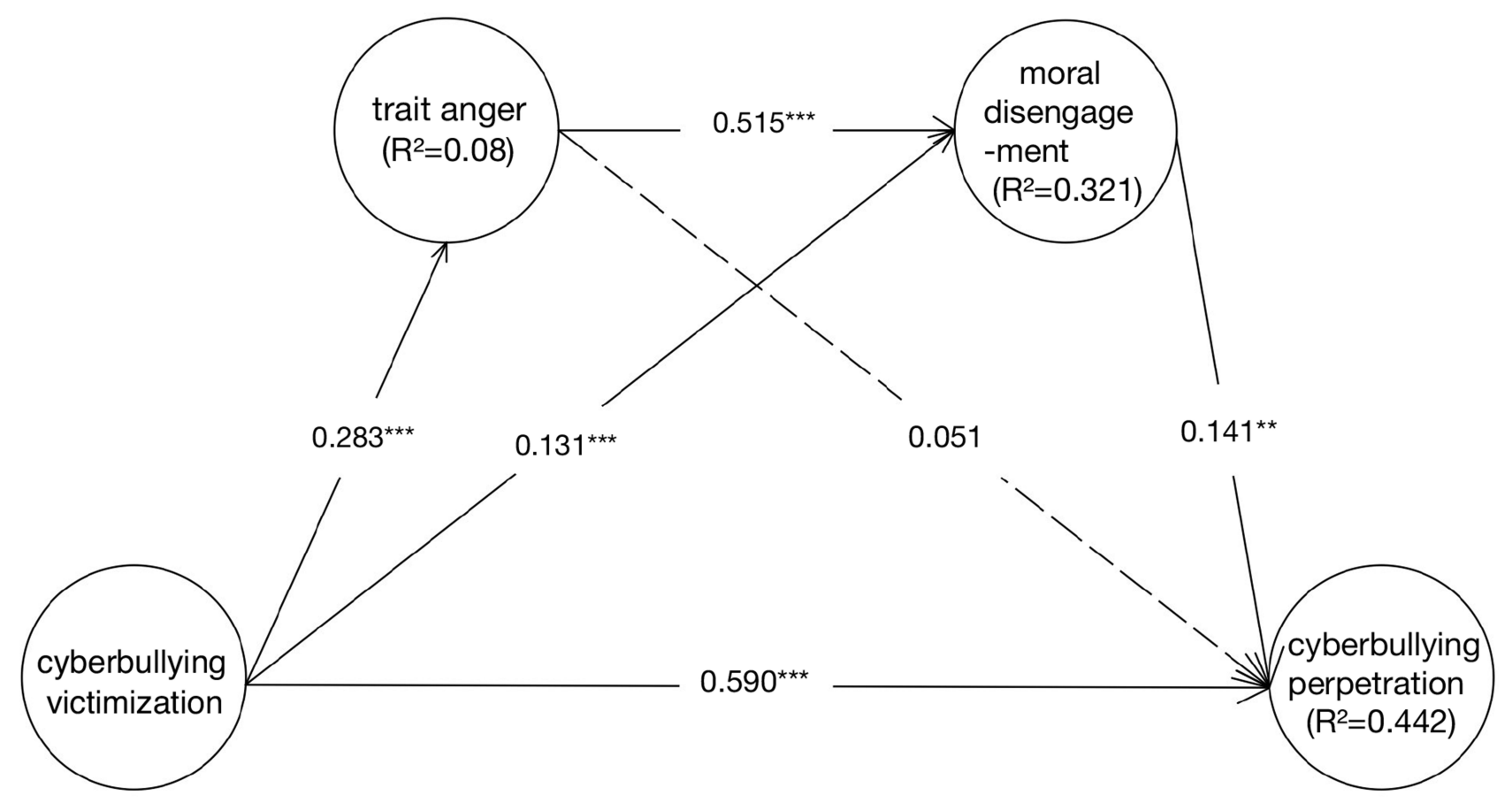

Figure 3 The model $B$ of the mediating effect.

Note: $*_{p}^{*}<0.01,{ }^{* * *} p<0.001$.

disengagement. Additionally, this could be influenced by cultural differences since individualistic cultures advocate expressing emotions, while collectivist cultures emphasize expressive suppression. Studies have found that expressive suppression is positively correlated with psychological problems, such as anxiety and depression, in individualistic cultures, but not in collectivist cultures. ${ }^{40-42}$ As an emotion regulation strategy, expressive suppression can regulate the relationship between social anxiety and cyberbullying perpetration. ${ }^{43}$ Future studies should be conducted to further investigate the moderating role of emotion regulation strategies in this model.

Hypothesis 3 of this study proposed that moral disengagement would separately mediate the relationship between cyberbullying victimization and perpetration. This study found that moral disengagement mediated the relationship between cyberbullying victimization and

Table 3 Fitting Indexes of Models

\begin{tabular}{|l|l|l|l|l|l|l|}
\hline Models & $\chi \mathbf{2}$ & $\mathbf{d f}$ & TLI & CFI & RMSEA & SRMR \\
\hline Model A & $28.646 * * *$ & 4 & 0.977 & 0.991 & 0.072 & 0.022 \\
Model B & $812.450 * * *$ & 84 & 0.895 & 0.916 & 0.086 & 0.044 \\
\hline
\end{tabular}

Note: $* * * p<0.001$

Abbreviations: TLI, Tucker-Lewis index; CFI, comparative fit index; RMSEA, rootmean-square error of approximation; SRMR, standardized root-mean-square residual; $\chi 2$, chi-square; $d f$, degree of freedom. perpetration. The more frequently individuals are bullied, the more likely they are to engage in cyberbullying. When individuals are bullied online, they are often under immense pressure and believe that there is a lack of supervision in the network. This establishes the belief that if they can be bullied by strangers online, they can also bully others at will. Moral disengagement, as an unhealthy cognitive mechanism, causes changes in individual cognition, reduces moral consciousness, and leads to unethical decisions and bad behavior. ${ }^{5,44,45}$ Therefore, the current results support Hypothesis 3.

\section{Research Limitations and Prospects}

This study has some limitations. First, cross-sectional studies cannot explain the causal relationship between variables; thus, longitudinal studies are required to explore the mechanism of the transformation from cyberbullying

Table 4 Bootstrap Analysis of Mediating Effect Test

\begin{tabular}{|l|l|l|}
\hline Model Pathways & $\begin{array}{l}\text { Standardized Indirect } \\
\text { Effect Estimation }\end{array}$ & $\begin{array}{l}\mathbf{9 5 \%} \mathbf{~ C l} \\
\text { Lower } \\
\text { Upper }\end{array}$ \\
\hline $\mathrm{CV} \rightarrow \mathrm{MD} \rightarrow \mathrm{CP}$ & $0.131 \times 0.141=0.018$ & 0.0060 .049 \\
$\mathrm{CV} \rightarrow \mathrm{TA} \rightarrow \mathrm{MD} \rightarrow \mathrm{CP}$ & $0.283 \times 0.515 \times 0.14 \mathrm{I}=0.02 \mathrm{I}$ & 0.0080 .047 \\
\hline
\end{tabular}

Abbreviations: $\mathrm{CV}$, cyberbullying victimization; TA, trait anger; MD, moral disengagement; $\mathrm{CP}$, cyberbullying perpetration. 
victimization to cyberbullying perpetration. Second, the sample for this study is from only one university, which may affect the generalizability of the results. In the future, diverse samples (such as from the field of sports, art, and medicine) should be studied. Third, although this study examines the roles of emotional and cognitive factors in the relationship between cyberbullying victimization and perpetration, how cyberbullying is influenced by external factors (cyber anonymity) or other individual factors (such as personality traits) needs to be studied further. Fourth, as cyberbullying victimization is closely related to cyberbullying perpetration, it indicates that cyberbullying may result from a vicious circle. However, this study only focuses on the impact of cyberbullying victimization on cyberbullying. Future studies can be conducted to explore the influence of cyberbullying perpetration on cyberbullying victimization and can compare the similarities and differences of the two influence mechanisms to analyze the cyclic processing model of cyberbullying. Fifth, according to the subtypes of cyberbullying, future research models should separately test for visual and written cyberbullying. Sixth, the study relied entirely on self-report questionnaires, which increases the risk of social desirability. ${ }^{46}$ Future studies should consider using interviews to test the effects of individual factors in the mechanisms of cyberbullying.

\section{Conclusions}

This study explored the mechanisms for the association between cyberbullying victimization and perpetration and examined the direct impact of cyberbullying victimization on cyberbullying perpetration and the indirect simultaneous influence of trait anger (emotional process) and moral disengagement (cognitive process). Results indicated that moral disengagement mediated the relationship between cyberbullying victimization and perpetration, and trait anger indirectly mediated this relationship through moral disengagement. Our findings suggest areas where college counselors could actively prevent cyberbullying through university intervention programs that improve students' moral cognition and behaviors, not only in the context of explicit aggression but also in cyberspace.

\section{Acknowledgments}

We would like to thank the editor and the anonymous reviewers for their useful comments on earlier drafts.

\section{Funding}

This research was supported by Liaoning Social Science Planning Foundation (Grant No. L20ASH005); The Scientific Research Fund Project of Education Department of Liaoning Province (Grant No. 2020LNQN08); University of Science and Technology Liaoning Talent Project Grants (601011507-33) awarded to Liang Chen, and supported by the Scientific Research Fund Project of Education Department of Liaoning Province (WQN2020ST09) awarded to Guangbo Dou.

\section{Disclosure}

The authors report no conflicts of interest in this work.

\section{References}

1. Tokunaga RS. Following you home from school: a critical review and synthesis of research on cyberbullying victimization. Comput Hum Behav. 2010;26(3):277-287. doi:10.1016/j.chb.2009.11.014

2. Menesini E, Nocentini A, Calussi P. The measurement of cyberbullying: dimensional structure and relative item severity and discrimination. Cyberpsychol Behav Soc Netw. 2011;14(5):267-274. doi:10.1089/cyber.2010.0002

3. Smith PK, Mahdavi J, Carvalho M, et al. Cyberbullying: its nature and impact in secondary school pupils. J Child Psychol Psychiatry. 2008;49(4):376-385. doi:10.1111/j.1469-7610.2007.01846.x

4. Slonje R, Smith PK. Cyberbullying: another main type of bullying? Scand $J$ Psychol. 2008;49(2):147-154. doi:10.1111/j.14679450.2007.00611.x

5. Thomas HJ, Connor JP, Scott JG. Integrating traditional bullying and cyberbullying: challenges of definition and measurement in adolescents - a review. Educ Psychol Rev. 2015;27(1):135-152. doi:10.1007/s10648-014-9261-7

6. Kraft E, Wang J. An exploratory study of the cyberbullying and cyberstalking experiences and factors related to victimization of students at a public liberal arts college. Int J Tech. 2011;1:74-91.

7. Zhu H, Shi FC, An L, et al. Investigation and analysis on the current situation of some college students' cyberbullying behavior in China. J Jilin Univ. 2016;42:605-611.

8. Gibb ZG, Devereux PG. Who does that anyway? Predictors and personality correlates of cyberbullying in college. Comput Hum Behav. 2014;38:8-16. doi:10.1016/j.chb.2014.05.009

9. Fang J, Wang X, Wen Z, Huang J. Cybervictimization and loneliness among Chinese college students: a moderated mediation model of rumination and online social support. Child Youth Serv Rev. 2020;115:105085. doi:10.1016/j.childyouth.2020.105085

10. Zhao BB, Jin CC, Wu YT. Influence of family function on cyberbullying among teenagers: chain mediation effect analysis. J Clin Psychol. 2018;26:102-107.

11. Ş A, Özdemir Y, Kuzucu Y. Cybervictimization and cyberbullying: the mediating role of anger, don't anger me! Comput Hum Behav. 2015;49:437-443. doi:10.1016/j.chb.2015.03.030

12. Patchin JW, Hinduja S. Traditional and nontraditional bullying among youth: a test of general strain theory. Youth Soc. 2011;43 (2):727e751. doi:10.1177/0044118X10366951

13. Lianos H, Mcgrath A. Can the general theory of crime and general strain theory explain cyberbullying perpetration? Crime Delinquency. 2017;64(5):674-700. doi:10.1177/0011128717714204 
14. Ybarra ML, Mitchell KJ. Online aggressor/targets, aggressors, and targets: a comparison of associated youth characteristics. J Child Psychol Psychiatry. 2004;45(7):1308-1316. doi:10.1111/j.14697610.2004.00328.x

15. König A, Gollwitzer M, Steffgen G. Cyberbullying as an act of revenge? Aust J Guid Couns. 2010;20(2):210-224. doi:10.1375/ajgc.20.2.210

16. Volk A, Craig W, Boyce W, King M. Adolescent risk correlates of bullying and different types of victimization. Int $J$ Adolesc Med. 2006;18:575-586.

17. Wong DSW, Chan HC, Cheng CHK. Cyberbullying perpetration and victimization among adolescents in Hong Kong. Child Youth Serv Rev. 2014;36:133-140. doi:10.1016/j.childyouth.2013.11.006

18. Hamer AHD, Konijn EA, Keijer MG. Cyberbullying behavior and adolescents use of media with antisocial content: a cyclic process model. Cyberpsychol Behav Soc Netw. 2014;17(2):74-81. doi:10.1089/cyber.2012.0307

19. Agnew R. Foundation for a general strain theory of crime and delinquency. Criminology. 1992;30(1):47-88. doi:10.1111/j.17459125.1992.tb01093.x

20. Gabriel RP. Cyberbullying among adolescents: a general strain theory perspective. $J$ Sch Violence. 2018;17(1):74-85. doi:10.1080/ 15388220.2016.1220317

21. Spielberger CD. State-Trait Anger Expression Inventory-2. Professional Manual. Odessa, FL: Psychological Assessment Resource; 1999:1-10.

22. Wang X, Yang L, Yang J, et al. Trait anger and cyberbullying among young adults: a moderated mediation model of moral disengagement and moral identity. Comput Hum Behav. 2017;73:519-526. doi:10.1016/j.chb.2017.03.073

23. Helfenfinger G. Moral disengagement and aggression: comments on the special issue. Merrill Palmer Quart. 2015;61(1):192-211. doi:10.13110/merrpalmquar1982.61.1.0192

24. Bandura A, Barbaranelli C, Caprara GV, Pastorelli C. Mechanisms of moral disengagement in the exercise of moral agency. J Pers Soc Psychol. 1996;71(2):364-374. doi:10.1037/0022-3514.71.2.364

25. Zhu XW, Zhou ZK, Xie HP, Zhu XW. Social expectation and evaluation of teachers' ethics in primary and secondary schools-empirical investigation based on audience perspective. J Beijing Normal Univ. 2019;271:54-59.

26. Erdur-Baker O, Kavsut F. Cyberbullying: a new face of peer bullying. Eurasian J Educ Res. 2007;27:31-42.

27. Zhou Z, Tang H, Tian Y, et al. Cyberbullying and its risk factors among Chinese high school students. School Psychol Int. 2013;34 (6):630-647. doi:10.1177/0143034313479692

28. Luo YL, Zhang DJ, Liu YB, Liu YL. Reliability and validity of the Chinese version of trait anger scale in college students. Chin Ment Health J. 2011;9:700-704.

29. Detert JR, Treviño LK, Sweitzer VL. Moral disengagement in ethical decision making: a study of antecedents and outcomes. $J$ Appl Psychol. 2008;93(2):374-391. doi:10.1037/0021-9010.93.2.374

30. Wang XC, Yang JPA. Study on the reliability and validity of the chinese moral shirking questionnaire. $J$ Clin Psychol. 2010;18:177-179.
31. Wright MF. Longitudinal investigation of the associations between adolescents' popularity and cyber social behaviors. J Sch Violence. 2014;13(3):291-314. doi:10.1080/15388220.2013.849201

32. Wang X, Lei L, Yang J, et al. Moral disengagement as mediator and moderator of the relation between empathy and aggression among Chinese male juvenile delinquents. Child Psychiatry Hum D. 2016;48:1-11.

33. Muthe'n LK, Muthe'n BO. Mplus User's Guide. 7th ed. Los Angeles, CA: Muthe'n \& Muthe'n; 1998-2012.

34. Wu Y, Wen ZL. Item parceling strategies in structural equation modeling. Adv Psychol Sci. 2011;19:1859-1867.

35. Cuevas CA, Finkelhor D, Turner HA, Ormrod RK. Juvenile victimization and delinquency: a theoretical typology. J Interpers Violence. 2007;22(12):1581-1602. doi:10.1177/0886260507306498

36. Law DM, Shapka JD, Hymel S, Olson BF, Waterhouse T. The changing face of bullying: an empirical comparison between traditional and internet bullying and victimization. Comput Hum Behav. 2012;28(1):226-232. doi:10.1016/j.chb.2011.09.004

37. Linton DK, Power JL. The personality traits of workplace bullies are often shared by their victims: is there a dark side to victims? Pers Individ Differ. 2013;54(6):738-743. doi:10.1016/j.paid.2012.11.026

38. Valkenburg PM, Peter J. Online communication among adolescents: an integrated model of its attraction, opportunities, and risks. $J$ Adolesc Health. 2011;48(2):121-127. doi:10.1016/j.jadohealth.2010.08.020

39. Bane CM, Cornish M, Erspamer N, Kampman L. Self-disclosure through weblogs and perceptions of online and " real-life" friendships among female bloggers. Cyberpsychol Behav Soc Netw. 2010;13(2):131-139. doi:10.1089/cyber.2009.0174

40. Liu W, Chen L, Blue PR. Chinese adaptation and psychometric properties of the child version of the cognitive emotion regulation questionnaire. PLoS One. 2016;11(2):e0150206. doi:10.1371/journal. pone.0150206

41. Liu W, Chen L, Tu X. Chinese adaptation of emotion regulation questionnaire for children and adolescents (ERQ-CCA): a psychometric evaluation in Chinese children. Int $J$ Psychol. 2017;52(5):398-405. doi:10.1002/ijop.12233

42. Liu W, Liu F, Chen L, et al. Cognitive reappraisal in children: neuropsychological evidence of up-regulating positive emotion from an ERP study. Front Psychol. 2019;10:147. doi:10.3389/fpsyg.2019.00147

43. Wang QQ, Fang CY, Chu XW. The relationship between adolescent cybervictimization and cyberbullying: a moderated mediated model. Dev Psychol. 2020;36:216-227.

44. Kowalski RM, Giumetti GW, Schroeder AN, Lattanner MR. Bullying in the digital age: a critical review and meta-analysis of cyberbullying research among youth. Psychol Bull. 2012;140(4):1073. doi:10.1037/ a0035618

45. Gini G, Pozzoli T, Bussey K. The role of individual and collective moral disengagement in peer aggression and bystanding: a multilevel analysis. J Abnorm Child Psychol. 2015;43(3):441-452. doi:10.1007/ s10802-014-9920-7

46. Dicken C. Good impression, social desirability, and acquiescence as suppressor variables. Educ Psychol Meas. 1963;43(4):699-720. doi:10.1177/001316446302300406
Psychology Research and Behavior Management

\section{Publish your work in this journal}

Psychology Research and Behavior Management is an international, peer-reviewed, open access journal focusing on the science of psychology and its application in behavior management to develop improved outcomes in the clinical, educational, sports and business arenas. Specific topics covered in the journal include: Neuroscience, memory and decision making; Behavior modification and management; Clinical applications; Business and sports performance management; Social and developmental studies; Animal studies. The manuscript management system is completely online and includes a very quick and fair peer-review system, which is all easy to use. Visit http://www. dovepress.com/testimonials.php to read real quotes from published authors. 